\title{
Laboreal
}

Volume $12 \mathrm{~N}^{\circ} 2$ | 2016

Equipamentos de Proteção Individual II

\section{Diseño de marcos para actuar y hacer actuar: la actividad de prescripción en una empresa hortícola}

Conceber quadros para agir e fazer agir: a atividade de prescrição em uma empresa de horticultura

Concevoir des cadres pour agir et faire agir : le travail de prescription dans une entreprise horticole

Lead the staff to act and make action: the prescription activity in a horticulture company

\section{Corinne Gotteland}

\section{OpenEdition}

\section{Journals}

Edición electrónica

URL: http://journals.openedition.org/laboreal/2688

DOI: $10.4000 /$ laboreal.2688

ISSN: 1646-5237

\section{Editor}

Universidade do Porto

Referencia electrónica

Corinne Gotteland, « Diseño de marcos para actuar y hacer actuar: la actividad de prescripción en una empresa hortícola », Laboreal [En línea], Volume 12 №2 | 2016, Publicado el 01 diciembre 2016, consultado el 24 septiembre 2020. URL : http://journals.openedition.org/laboreal/2688 ; DOI : https:// doi.org/10.4000/laboreal.2688

Este documento fue generado automáticamente el 24 septiembre 2020.

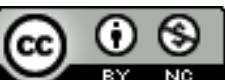

Laboreal está licenciado com uma Licença Creative Commons - Atribuição-NãoComercial 4.0 Internacional. 


\section{Diseño de marcos para actuar y hacer actuar: la actividad de prescripción en una empresa hortícola}

Conceber quadros para agir e fazer agir: a atividade de prescrição em uma empresa de horticultura

Concevoir des cadres pour agir et faire agir : le travail de prescription dans une entreprise horticole

Lead the staff to act and make action: the prescription activity in a horticulture company

\section{Corinne Gotteland}

\section{REFERENCIA}

Gotteland-Agostini, C. (2013). Concevoir des cadres pour agir et faire agir : le travail de prescription dans une entreprise horticole. Thèse de doctorat en Ergonomie, préparée au Créapt, S. Volkoff et V. Pueyo (dir.), Cnam, CEE, Noisy-le-Grand.

\section{NOTA DEL EDITOR}

http://dx.doi.org/10.15667/laborealxii0216cg

Manuscrito recibido en: noviembre/2015

Aceptado tras peritaje: junio/2016

1 Esta tesis, desarrollada en el marco del proyecto AgriQuadra [1], se refiere a la actividad de prescripción en una empresa del sector hortícola perteneciente a un grupo de 
productores franceses. Estamos particularmente interesados en la actividad de elaboración de las prescripciones realizadas por supervisores encargados de prescribir y organizar el trabajo de los obreros de producción (Gotteland-Agostini, 2013).

\section{El trabajo de los supervisores desde el punto de vista de la ergonomía}

2 En ergonomía, varios estudios se han concentrado en enseñar las especificidades de la actividad de los supervisores en las grandes empresas (Langa, 1994, Carballeda, 1997; Mascia, 1994, 2001). El supervisor aparece como un operador en condiciones de trabajo especifico en la interface de limitaciones ascendente y descendentes debido a su posición jerárquica intermediaria en la empresa. En el sector de la construcción, algunos autores se centraron en los aspectos organizativos de la producción (Bergamini, 1995; Bergamini y Beguin,1996); otros observaron las evoluciones profesionales relacionadas con la gestión procedimientos (Six y Tracz, 1997); otros aun analizaron las condiciones de elaboración de las prescripciones destinadas a mejorar la prevención de riesgos (Six, 1999). Más recientemente, estudios que se centraron en la dimensión cognitiva de la actividad de los supervisores en la aeronáutica (Lebris, 2010) y en la construcción (Forrière, Anceaux, Cégerra y Six, 2011), dieron testimonio del creciente interés de los ergónomos en estos trabajadores. En horticultura, Zara-Meylan (2012) ha demostrado que su actividad consiste en la gestión de la incertidumbre y de las múltiples limitaciones de tiempo. Independientemente de su situación y función, todos estos estudios subrayan la complejidad del trabajo de los supervisores, ya que tienen que llevar a cabo tareas múltiples y variadas: de relaciones, de gestión, comerciales y técnicas (Mascia, 2001). Otros estudios tratan las cuestiones relacionadas con el cotidiano de los supervisores que enfrentan dificultades de gestión del desempeño (Piney, Nascimento, Gaudart y Volkoff, 2014) o de la necesidad de un marco formal para el ejercicio de la confianza (Van Belleghem, 2013). Pocos estudios en ergonomía tratan el trabajo de los supervisores, tema al que nos dedicamos, con el fin de enriquecer con aportaciones teóricas, centrando nuestro análisis en una faceta de su trabajo: la elaboración de prescripciones relacionada con la organización de la producción.

\section{La elaboración de la prescripción}

3 En el campo léxico, las prescripciones evidencian diferentes lógicas relacionadas con el actuar. Que es a la vez una acción de quien elabora una prescripción y un soporte de acción para los destinatarios, pudiendo, así, ser una limitación o un recurso para la acción. En esta tesis, consideramos la prescripción tanto como la acción de un proscriptor como el resultado de la acción del mismo, que dirige a uno o más destinatarios.

4 Una acción es un proceso que conduce a un resultado; creemos que una prescripción es un proceso que conduce a un producto - un "artefacto prescriptivo" llamado él también de prescripción. Para analizar el proceso de elaboración de la prescripción, se considera que la actividad de la persona que prescribe también es una actividad de diseño (Falzon, 1995) desarrollada tal y como un modelo de diseño (Béguin, 2010). En el caso estudiado, 
el proyecto del supervisor que elabora las prescripciones es lograr "completar todo el trabajo que hay que hacerse" con los medios disponibles (recursos internos y externos, trabajadores disponibles), en un día de trabajo, o incluso antever varios días consecutivos (p. ej., empezar a trabajar un día y terminar unos días después).

\section{El trabajo de gestión visto como una actividad de diseño}

5 El trabajo de gestión puede ser comprendido como una actividad de diseño durante la cual el supervisor diseña "marcos de acción" (Gotteland-Agostini, 2013). Según Goffman (1974), todo individuo moviliza esquemas interpretativos que el autor nombra de "marcos primarios" (naturales y sociales) movilizados para definir la manera de actuar más adecuada frente a una dada situación. Sin embargo, estos marcos primarios no son suficientes para producir una acción bien ajustada. El actor está entonces en un "dialogo con la situación" en los términos de Schön (1983). El actor conoce a contingencias situacionales: resistencias que debe tomar en consideración $u$ oportunidades que puede coger para actuar (Joas, 2004) y que lo conducen, según los términos de Goffman (op. cit.), a producir "marcos secundarios". El objetivo de esta tesis es caracterizar "los marcos primarios" existentes y analizar la actividad de diseño de las "adaptaciones secundarias" producidas por un supervisor del sector hortícola.

6 La empresa en la que se desarrolló esta investigación, tiene unos veinte empleados permanentes y otros tantos de temporada durante la temporada alta (de marzo a junio), cuando las operaciones de producción se acumulan con las ventas de plantas perennes, anuales y de temporada (Agostini, 2007). Esta situación presenta dos importantes dimensiones. En primer lugar, la producción se lleva a cabo en un entorno dinámico y abierto, característico del sector hortícola (Zara-Meylan, 2012). La incertidumbre asociada con el elemento natural es preponderante (el clima, los productos vivos), así como las reglas de agricultura, de botánica y sanitarias necesarias para llegar a producir plantas sanas y de calidad. En segundo lugar, la empresa forma parte de un grupo francés de productores con tres grupos regionales que imponen sus propias reglas heterónomas (Petit, 2005) o externas a la empresa. Ese funcionamiento en grupo condiciona la elaboración de normas endógenas (Petit, op. Cit.) o internas en la empresa. Todas estas características incluyen los elementos constitutivos en los marcos primarios (naturales y sociales) que preexisten al trabajo del supervisor. Estos son los "marcos para actuar" en los que el supervisor se va a apoyar para desarrollar las adaptaciones secundarias o los "marcos de acción" destinados, entre otros, a orientar a los equipos de trabajo bajo su responsabilidad.

\section{Un enfoque metodológico para comprender la actividad de prescripción del supervisor}

Con el objetivo de comprender la actividad de elaboración de la prescripción del supervisor, el enfoque metodológico se basa en el Análisis Ergonómico del Trabajo (Wisner, 1994) para distinguir los marcos primarios (naturales y sociales) preexistentes a partir del grupo y de la empresa, y los marcos secundarios elaborados: los para actuar (p. ej., para pensar la situación, para él - su planificación de trabajo - y para los demás - 
la afectación de los equipos) y los para hacer actuar (p. ej., para ayudar en la realización de los trabajos de producción y órdenes de pedido, para los demás).

8 Las observaciones de la actividad de prescripción del supervisor 嘔河 aportan una diversidad de situaciones relacionadas con la organización de la producción. Considerando ese responsable como "operador común", lo hemos seguido durante los

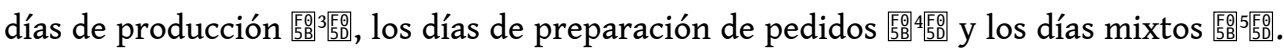
Las observaciones sistemáticas se realizaron en once jornadas de trabajo completas de 8 h00 a 17h00, a veces incluso después de este horario, los lunes, cuando había muchos pedidos, ya que se deberían preparar todas las plantas en el mismo día. En el periodo observado, los equipos bajo el mando del supervisor estaban compuestos por doce trabajadores permanentes y catorce trabajadores de temporada (es el número de trabajadores de temporada que el supervisor contrató en las últimas cinco temporadas).

9 Para analizar su actividad de prescripción, se registraron durante horas sus acciones, sus interlocuciones, sus desplazamientos, etc... Grabamos sus intercambios verbales con cada uno de sus colegas (superiores jerárquicos y subordinados) para identificar las prescripciones que recibe y emite, además de los comentarios espontáneos hechos en el curso de su acción de preparación del trabajo (planificación, notas manuscritas, etc). Nuestro objetivo era recoger elementos sobre los momentos de prescripción (razón, forma, contenido, intercambios, etc). A continuación, transcribimos y organizamos los datos que recogimos, para obtener el desarrollar de su actividad en cada uno de los días observados.

Del conjunto de estos elementos, se realizaron entrevistas de explicación y auto confrontaciones (Mollo, 2004) $(\mathrm{n}=27)$ con una duración aproximada de $1 \mathrm{~h} 15$ a 2 horas cada una, realizadas a partir de rasgos de su actividad obtenidos durante las observaciones (planificaciones, notas manuscritas), y sobre los tiempos dedicados exclusivamente a la elaboración y a la transmisión de planificaciones, instrucciones orales y manuscritas. Como es la norma en la ergonomía de la actividad, sus comentarios se incluyeron en el análisis y nos permitieron clarificar y enriquecer los resultados.

11 Además, pusimos de relieve de manera exhaustiva las prescripciones existentes vinculadas a la actividad de los supervisores (lo que reciben y lo que emiten). Con base en la literatura, se identificaron los criterios (p. ej., contenido, propósito, transmisor, receptor, etc.) para distinguir los diferentes tipos de prescripciones.

12 Los principales resultados obtenidos con este análisis de la prescripción se refieren a la caracterización de los marcos para la acción y a la actividad de diseño de eses marcos de acción.

\section{La actividad de prescripción del supervisor: transmitir o adaptar los marcos para la acción y diseñar los marcos de acción}

13 El supervisor utiliza tres modalidades para prescribir el trabajo: (i) transmite tal cual o (ii) adapta los marcos primarios naturales y sociales (para los demás) y diseña marcos secundarios para actuar y hacer actuar (para sí mismo y para los demás) (GottelandAgostini, 2013). En este tercer caso, el diseño de los marcos secundarios se produce por 
la conducta de micro proyectos diarios u ocasionales o de secuencias de diseño, como está ilustrado en la figura 1.

Figura 1: la actividad de prescripción del supervisor

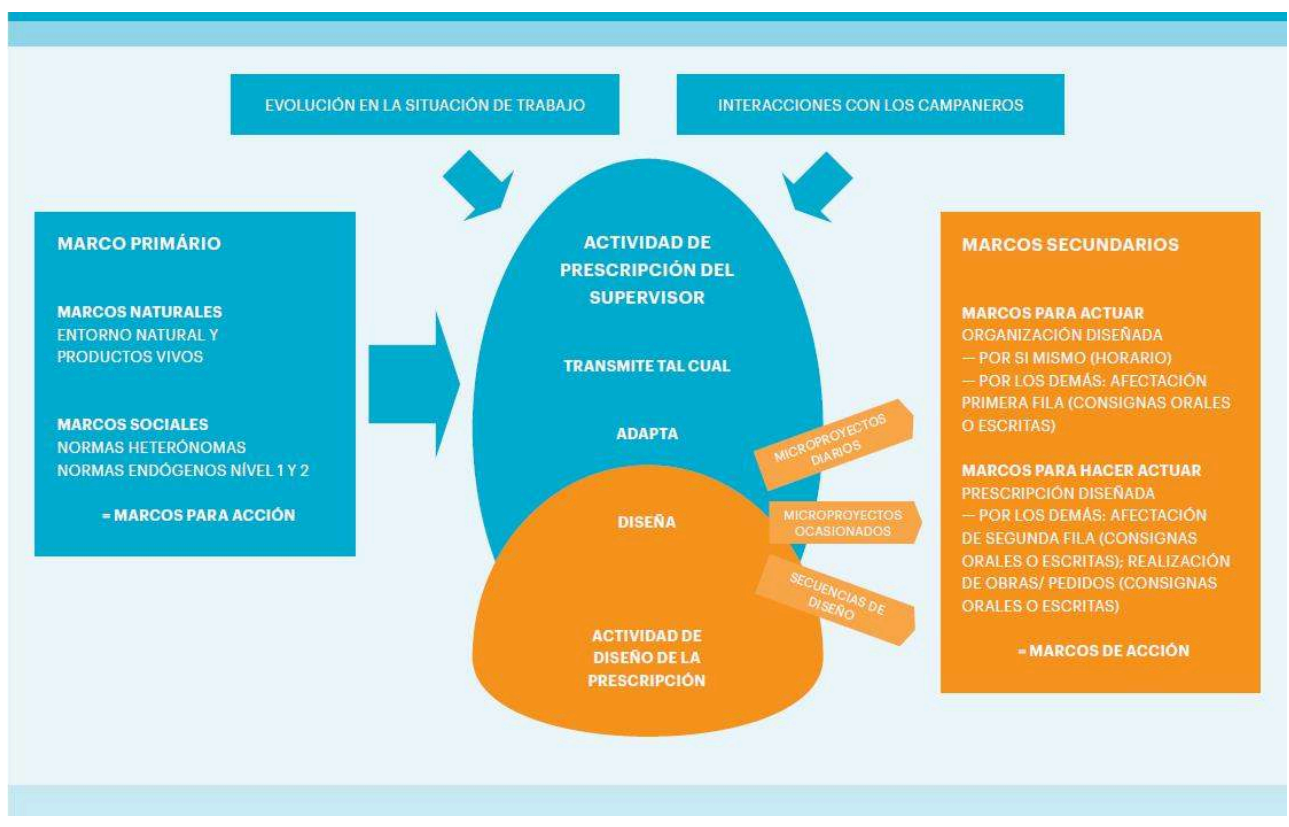
(empleados actuales, condiciones meteorológicas, las obras, las plantas, etc..) y las diversas interacciones con los subordinados, compañeros, jefes y clientes. Luego monitorea el "hacer hacer", coordinando el trabajo para garantir su realización y el "hacer" de los trabajadores, los que están encargados de la realización en el campo de producción o en las ventas de productos.

17 Es importante añadir que los marcos de acción no se estabilizan una vez por todas. Según las interacciones, las variabilidades naturales e industriales, el supervisor puede ser llevado a reconsiderar los marcos de acción para sí mismo y para los demás (tanto los marcos de actuar como los para hacer actuar). Esta nueva movilización de los marcos se produce mediante acciones de "enmarque", "re-enmarque" y de "co- 
enmarque", siempre inscribiéndose en "micro proyectos" o en "secuencias de diseño" (Gotteland-Agostini, 2013).

\section{La conducta de micro proyectos y de secuencias de diseño}

Los micros proyectos diarios se conducen con o sin el apoyo de una planificación. El objetivo es organizar "todo" el trabajo que hay que hacer en el día (emergencias relacionadas al vegetal, trabajos de producción, preparación y envío de pedidos, etc), definiendo marcos para actuar para sí mismo y para los demás. Los resultados muestran que las acciones de "re-enmarque"realizadas al comienzo del día y que conducen a un rediseño integral de la planificación y de las acciones de "enmarque" realizadas a lo largo del día permiten continuar con el diseño de los marcos de acción en la medida en que el trabajo avance.

Los micro proyectos ocasionales se imponen a todos los trabajadores que hay que gestionar el mismo día o que necesitan una preparación previa (pedidos de cantidades importantes, por ejemplo). Aquí, el objetivo es repartir el trabajo que hay que hacer (marcos para la acción) y que hay que "hacer hacer" (marco de acción) a lo largo de varios días para estar listo en "el día final" (entrega). En este caso, se observan las acciones de "co-enmarque" entre el supervisor y sus colegas de cualquier nivel jerárquico.

Las secuencias de diseño se construyen según sea necesario y poco a poco, lo que permite la elaboración de marcos para hacer actuar con el objetivo de facilitar la realización del trabajo que hay que hacer sobre el terreno (en las zonas de cultivo o de preparación de pedidos). Las acciones de co-enmarque han sido puestas en evidencia entre el supervisor y sus subordinados, revelando como ellas permitieron discutir colectivamente y durante la acción, para saber cómo proceder.

\section{No una prescripción, sino un marco para la acción y marcos de acción}

21 En esta empresa hortícola, la prescripción adopta formas múltiples. Su elaboración sigue circuitos diferenciados según los tiempos de funcionamiento de la empresa (producción y comercialización). En este haz de prescripciones, el supervisor ocupa un sitio central. Está en contacto con todos los interlocutores (internos y externos) de la empresa, mismo si no intercambia con los mismos interlocutores (excepto sus subordinados) durante los trabajos de producción y la preparación de los pedidos (mandos). El supervisor es el último eslabón en el circuito de elaboración de la prescripción, ya que está situado jerárquicamente justo por encima de los obreros.

Desde el punto de vista de los marcos primarios (o marcos para la acción), preexistentes al trabajo del supervisor, nuestros resultados muestran que algunas de sus prescripciones son presentes al nivel de dos circuitos: producción y comercialización producción. Además, el análisis de los diferentes criterios pone de manifiesto que los marcos primarios (o marcos para la acción) pueden ser negociables según su nivel de emisión. Así, en su elaboración de la prescripción, el supervisor observado puede volver a discutir las reglas endógenas (nivel local) así como algunas reglas heterónomas del 
nivel regional en la etapa de comercialización (p. ex., hora de paso de los camiones del grupo para recoger los pedidos). Sin embargo, no puede realizar ninguna acción sobre las reglas heterónomas al nivel nacional (p. ex., calendario de las ventas).

Desde el punto de vista de los marcos secundarios (o de los marcos de acción) concebidos por el supervisor: estos pueden ser analizados como acciones realizadas en un determinado instante. Por una parte, los marcos de acción son pocos y no reproducibles debido a la diversidad de las plantas trabajadas y de la variabilidad intrínseca de estos productos vivos, cuya calidad evoluciona sin cesar. Por otra parte, el supervisor concíbelos teniendo en cuenta la experiencia (conocimientos del vegetal y las competencias) de sus destinatarios (trabajadores de temporada novicios y trabajadores permanentes y experimentados).

\section{La actividad de prescripción del marco: una actividad de diseño a veces enmarcada y a veces "enmarcante"}

Nuestro análisis muestra que la actividad de diseño realizada por el supervisor consiste en la realización de micro proyectos y secuencias de diseño realizadas casi exclusivamente al oral en la acción y en la "confrontación con el real" de la situación (desarrollo del trabajo, intercambios con los colegas, las necesidades del vegetal, condiciones meteorológicas). Este proceso de diseño puede estar considerado como el resultado de una superposición de marcos de acción, para repetir los términos de Goffman (1974), à la estratificación de marcos secundarios.

Los artefactos producidos durante el proceso de diseño son útiles para organizar el trabajo. Es particularmente el caso de las planificaciones de trabajos cuyo uso queda exclusivamente reservado al supervisor. Sus planificaciones no siempre son materializadas, excepto cuando se "vuelven demasiado pesadas para guardar" mentalmente. Otros artefactos sirven al supervisor para prescribir el trabajo, como las orientaciones que son esencialmente orales y pocas veces manuscritas.

La actividad del supervisor es una actividad de diseño compleja desarrollada en condiciones de incertidumbre. En primer lugar, está enmarcada por los marcos preexistentes a su actividad (como la organización de la empresa y del grupo) y por la naturaleza del propio proceso de diseño. En realidad existe en la gestión de proyectos una irreversibilidad consecutiva a cada paso de validación que conduce a la solución final. Por otra parte, esta actividad es también "enmarcante" porque resulta en el diseño de marcos para actuar y para hacer actuar.

Por lo tanto, enmarcar no es solo transmitir o adaptar las prescripciones recibidas, sino que también es conducir micro proyectos y secuencias de diseño con intervalos de tiempo variados. Al mismo tiempo, enmarcar también es interactuar con personas situadas en diferentes niveles organizativos y jerárquicos, en función de los diferentes momentos de la organización de la producción.

Esta tesis demuestra la organización del trabajo de PME cerca de granjas agrícolas que tienen actividades específicas y sus propios problemas derivados de los cambios iniciados en los últimos años. Por lo tanto, puede considerarse como un aporte de conocimiento en ergonomía en el sector de la horticultura, sector muy poco estudiado.

En esta tesis también se presenta una visión de la actividad de diseño a partir de la realización de micro proyectos realizados en tiempo real y en respuesta a las 
resistencias emergentes de la situación de trabajo. Su objetivo es lograr en ese momento la prescripción más adecuada para que los trabajadores puedan actuar de la manera más eficaz posible. Además, esta tesis sugiere otra manera de considerar el concepto de actividad en ergonomía, a partir de la racionalidad no teleológica de acción, es decir apelando a la creatividad del actuar y de proyectos singulares.

\section{BIBLIOGRAFÍA}

Agostini, C. (2007). Liens entre prescription et organisation du travail : quelles conséquences pour la santé ? Unpublished Mémoire de Master Recherche. CNAM, Paris.

Béguin, P. (2010). Conduite de projet et fabrication collective du travail : une approche développementale. Document pour l'Habilitation à Diriger des Recherches, Université Victor Segalen Bordeaux 2.

Béguin, P., Bergamini, J.F. (1996). Organiser la conception pour le chantier. In R. Patesson (s/d) Intervenir par l'ergonomie, Actes du XXXI ${ }^{\text {ème }}$ Congrès de la Self. Bruxelles, Vol.1, pp. 219-223.

Bergamini J.F. (1995). Du virtuel au réel - Quelques aspects de l'activité de chef de chantier. Unpublished Mémoire de DEA d'Ergonomie. Paris, CNAM - EPHE.

Carballeda, G. (1997). La contribution des ergonomes à l'analyse et à la transformation de l'organisation du travail : l'exemple d'une intervention relative à la maintenance dans une industrie de process continu. Unpublished Thèse de Doctorat d'ergonomie. Paris: CNAM, Laboratoire d'Ergonomie.

Falzon, P. (1995). Les activités de conception : réflexions introductives. Performances Humaines et Techniques, 74, 7-11.

Forrière, J, Anceaux, F., Cégerra, J., Six, F. (2011). L'activité des conducteurs de travaux sur les chantiers de construction : ordonnancement et supervision d'une situation dynamique. Le Travail Humain, 74(3), 283-308.

Goffman, E. (1974). Les cadres de l'expérience. Paris: Les éditions de Midi.

Gotteland-Agostini, C. (2013). Concevoir des cadres pour agir et faire agir : le travail de prescription dans une entreprise horticole. Thèse de doctorat en Ergonomie, préparée au Créapt, S. Volkoff et V. Pueyo (dir.), Cnam, CEE, Noisy-le-Grand.

Joas, H. (2004). La créativité de l'agir. Paris: Edition du Cerf.

Langa, P. (1994). Adaptation ou création de l'organisation du travail lors d'un transfert de technologie - Analyse de l'activité de l'encadrement et conception de l'organisation. Unpublished Thèse de Doctorat en Ergonomie, CNAM Paris.

Le Bris, V. (2010). La continuité des activités de production dans des systèmes postés discontinus : approche ergonomique des activités de relève de poste de l'encadrement de proximité. Unpublished Thèse de Doctorat en Ergonomie, Université Toulouse le Mirail. 
Mascia, F.L. (1994). La gestion de la production, une approche ergonomique du travail du chef d'atelier. Unpublished Mémoire de D.E.A. d'Ergonomie. Paris: Laboratoire d'Ergonomie Physiologique et Cognitives, EPHE.

Mascia, F.L. (2001). Gérer dans et avec l'atelier : une approche ergonomique du travail de la maîtrise dans le secteur industriel de production à grande échelle. Unpublished Thèse de Doctorat en Ergonomie. Paris: Laboratoire d'Ergonomie Physiologique et Cognitive, EPHE.

Mollo, V. (2004) Auto- and allo- confrontation as tools for reflective activities. Applied Ergonomics, 35 (6), pp. 531-540.

Petit, J. (2005) Organiser la continuité du service : intervention sur l'organisation d'une mutuelle de santé. Thèse d'Ergonomie. Université Victor Segalen Bordeaux 2 - ISPED. Laboratoire d'Ergonomie des Systèmes Complexes.

Piney C., Nascimento A., Gaudart C., Volkoff S. (2013). « Entre indicateurs et travail réel : l'expérience de l'encadrement de proximité dans un service public ", Actes du $48^{\text {ème }}$ congrès, et $50^{\text {ème }}$ anniversaire, de la Self, Ergonomies et société : quelles questions, quelles réponses ?, 28-30 août, La Sorbonne, Paris.

Schön (1983) The reflective practitioner. How professionals think in action. 1 ed., New York, Basic books

Six, F. (1999). De la prescription à la préparation du travail : apports de l'ergonomie à la prévention et à l'organisation du travail sur les chantiers de bâtiment, Document pour l'Habilitation à Diriger des Recherches, Lille : Université Charles de Gaulle Lille 3.

Six, F. \& Tracz, C. (1997). L'encadrement de chantier : des évolutions sous le regard de l'ergonomie. Performances Humaines \& Techniques, 91, 25-29.

Van Belleghem, L. (2013). Réciprocité des enjeux de confiance au travail - Le cas des coursiers et de leur dispatcheur. In L. Karsenty (coord.), La confiance au travail. Toulouse : Octarès, P.53-75.

Wisner, A. (1994). La cognition et l'action située : conséquences pour l'A.E.T. et l'anthropotechnologie. Version Française de la Communication Plénière, Congrès de l'IEA, Toronto. Joas, H. (2004). La créativité de l'agir. Paris: Edition du Cerf.

Zara-Meylan, V. (2012). « Modalités de gestion du milieu temporel dans une conduite de processus multiples en situation dynamique : une recherche dans des entreprises horticoles ", Thèse de doctorat en Ergonomie, CNAM-CREAPT, Paris.

\section{NOTAS}

1. "AgriQuadra" es un proyecto de igualdad en los enlaces de envejecimiento y el trabajo en los sectores agrícolas, conducido en colaboración con los centros estatales de fondo de seguro de los empleados de granjas y empresas agrícolas (FAFSEA), el fondo central de seguridad social agrícola (CCMSA) y la federación nacional de productores de horticultura y viveros (FNPHV) de 2006 a 2008.

2. En esta empresa, el jefe de cultura no está encargado de la integridad del proceso productivo, a la diferencia de otros estudios realizados en el sector (Meylan, 2012). Aunque poco difundido, este recorte no es excepcional; lo encontramos entre otros productores del mismo grupo. De modo que, en cuanto las plantas salgan de la multiplicación para nuevas macetas, el seguimiento de su cultura y la organización de la producción dependen de este supervisor. Este supervisor tiene 37 anos.Tiene diez años de antigüedad y como todos los empleados, ha sido contratado como 
obrero de producción. Luego su formación bac+2 en horticultura le permitió acceder al cabo de 5 anos a la función de supervisor del seguimiento de las culturas y expediciones.

3. Días de trabajo dedicados únicamente a las instalaciones de producción (martes y miércoles).

4. Días de trabajo dedicados únicamente a la preparación de los pedidos (lunes).

5. Días de trabajo mixtos, estando la mañana (de $8 \mathrm{~h}$ a $11 \mathrm{~h}$ ) dedicada a las instalaciones de producción y la segunda parte del día (de 11h a 17h) dedicada a la preparación de pedidos.

\section{AUTOR}

\section{CORINNE GOTTELAND}

Environnement Ville et Société (EVS) - UMR5600, Université de Lumières Lyon 92, Rue Pasteur 69007 Lyon France

co.gotteland@gmail.com 